\title{
Neutral current neutrino-nucleus interactions at high energies
}

\author{
M. B. Gay Ducati, ${ }^{1}$ M. M. Machado, ${ }^{1}$ and M. V. T. Machado ${ }^{2}$ \\ ${ }^{1}$ High Energy Physics Phenomenology Group, GFPAE, IF-UFRGS, Caixa Postal 15051, CEP 91501-970, Porto Alegre, Rio Grande do \\ Sul, Brazil \\ ${ }^{2}$ Centro de Ciências Exatas e Tecnológicas, Universidade Federal do Pampa, Campus de Bagé, \\ Rua Carlos Barbosa, CEP 96400-970, Bagé, Rio Grande do Sul, Brazil
}

(Received 7 January 2009; published 14 April 2009)

\begin{abstract}
We present a QCD analysis of the neutral current $(\mathrm{NC})$ neutrino-nucleus interaction at the small- $x$ region using the color dipole formalism. This phenomenological approach is quite successful in describing experimental results in deep inelastic ep scattering and charged current neutrino-nucleus interactions at high energies. We present theoretical predictions for the relevant structure functions and the corresponding implications for the total $\mathrm{NC}$ neutrino cross section. It is shown that at small $x$, the $\mathrm{NC}$ boson-nucleon cross section should exhibit the geometric scaling property that has important consequences for ultrahigh energy neutrino phenomenology.
\end{abstract}

DOI: 10.1103/PhysRevD.79.073008

PACS numbers: $13.15 .+\mathrm{g}, 13.60 . \mathrm{Hb}, 12.38 . \mathrm{Bx}$

\section{INTRODUCTION}

The interaction of high-energy neutrinos in hadron targets is an important probe to test quantum chromodynamics (QCD) and useful in understanding the parton properties of a hadron structure. Several combinations of neutrino and antineutrino scattering data can be used to determine the structure functions, which constrain the valence, sea, and gluon parton distributions in the nucleons or nuclei. In a similar way as for charged-lepton deep inelastic scattering (DIS), the deep inelastic neutrino scattering is also used to investigate the structure of nucleons and nuclei. In the leading order quark-parton model, the structure function $F_{2}$ is the singlet distribution, $F_{2}^{\nu N} \propto$ $x q^{S}=x \sum(q+\bar{q})$, the sum of momentum densities of all interacting quark constituents. These relations are further modified by higher order QCD corrections. Currently, the theoretical description of experimental data in neutrino DIS is reasonable, where main theoretical uncertainties are the role played by nuclear shadowing in contrast with lepton-charged DIS, and a correct understanding of the low $Q^{2}$ limit. However, nuclear effects are taken into account by using the nuclear ratios $R=F_{2}^{A} / A F_{2}^{p}$ extracted from lepton-nucleus DIS, which could be different for the neutrino-nucleus case. The low- $Q^{2}$ region cannot be addressed within the perturbative (pQCD) quark-parton model since a hard momentum scale $Q_{0}^{2} \geq 1-2 \mathrm{GeV}^{2}$ is required in order to perform perturbative expansion.

Concerning the important issues raised above, in Ref. [1] we performed an analysis of small- $x$ charged current (CC) neutrino-nucleus DIS using the color dipole formalism [2]. The structure functions $F_{2}^{\nu N}, x F_{3}^{\nu N}$, and the quantity $\Delta x F_{3}^{\nu N}$ were calculated and compared with the experimental data from CCFR [3-5] and NuTeV [6] by employing phenomenological parametrizations for the dipole cross section which successfully describe small- $x$ inclusive and diffractive $e p$ DIS data. In addition, we predicted the nuclear ratios $R_{2}$ and $R_{3}$ and singled out the size of nuclear effects in each case. It was found that small- $x$ data show geometric scaling property for the boson-hadron cross section, and the structure function $F_{2}$ is in agreement with the phenomenological implementation using the saturation models at the small- $x$ region [1]. The structure functions $x F_{3}^{\nu N}$ were discussed in detail, where the sea content described by the quantity $\Delta x F_{3}^{\nu N}$ is well described. This investigation calls attention to the robustness of the color dipole formalism to describe the total neutrino cross section of ultrahigh energy neutrinos at very high energies.

Our goal in this work is to perform similar investigation for neutrino neutral current (NC) scattering, which is an ideal probe for new physics. As an additional motivation to study NC neutrino interactions we quote the purpose of a new high-energy, ultrahigh statistics neutrino scattering experiment, NuSOnG (neutrino scattering on glass) [7]. This experiment uses a Tevatron-based neutrino beam to obtain an order of magnitude higher statistics than presently available for the purely weak $\nu_{\mu}+e^{-}$interactions, and DIS events which are about 2 orders of magnitude larger than past measurements will also be obtained. The very high statistics will permit an electroweak measurement using the DIS data sample from NuSOnG, through the "Paschos-Wolfenstein method" [8]. The best electroweak measurement using DIS events to date comes from the NuTeV experiment, which has observed an anomaly. The DIS measurement requires the knowledge of parton distribution functions which describe the momentum distribution of quarks as a function of $Q^{2}$ and can bring in theoretical uncertainties from sources such as nuclear effects and nuclear isospin violation. NuSOnG probably will improve statistics in comparison to the previous highest statistics experiments NuTeV and CCFR. Theoretical uncertainties can be addressed by making a high statistics measurement of the parton distribution functions on glass, in situ. NuSonG will generate an unprecedented sample of 
$>100$ M DIS events which can be used to measure six structure functions (three on neutrinos and three for antineutrinos) as well as the strange and antistrange parton distributions. The NuSOnG experiment intends to measure separately $\Delta F_{2} \equiv \frac{5}{18} F_{2}^{\mathrm{CC}}\left(x, Q^{2}\right)-F_{2}^{\mathrm{NC}}\left(x, Q^{2}\right)$ in $\mathrm{CC} W^{ \pm}$ exchange and $\mathrm{NC} \gamma / Z$ exchange processes, which can help to constrain charge symmetry violation.

In this work we present a determination of the small- $x$ structure functions for a NC neutrino nucleus within the color dipole formalism. This approach allows for a simple implementation of shadowing corrections [9] in neutrinonuclei interactions. This paper is organized as follows. In Sec. II, the structure functions $F_{2}^{\nu N}, F_{L}^{\nu N}$, and the nuclear ratio $R_{A}=F_{2}^{\nu A} / A F_{2}^{\nu N}$ are investigated in this approach at the small- $x$ region, employing recent phenomenological parton saturation models. It is shown that at small $x$, the $\mathrm{NC}$ boson-nucleon cross section should exhibit the geometric scaling property that has important consequences for ultrahigh energy neutrino phenomenology. Finally, we also analyze the charm contribution to the total $\mathrm{NC}$ neutrino-nucleus cross section. The prediction is consistent with the existing experimental determinations. In the last section we summarize the results and present our conclusions.

\section{NEUTRAL CURRENT NEUTRINO-NUCLEON INTERACTION AT HIGH ENERGIES}

In the color dipole approach [2], the small- $x$ DIS is treated in terms of the interaction of the $q \bar{q}$ color dipole of size $r$ with the target nucleon (or nucleus) which is characterized by the beam/flavor independent color dipole cross section. The color dipole formalism allows an all twist computation, in contrast with the usual leading twist approximation of the structure functions. In the neutral current neutrino interaction, the process $Z^{0}+N \rightarrow X$ is viewed as the result of the interaction of a color singlet dipole, in which the gauge boson $Z^{0}$ fluctuates into, with the nucleon target. The interaction is modeled via the dipole-target cross section, whereas the boson fluctuation in a color dipole is given by the corresponding light-cone wave function. The NC DIS structure functions [10-12] are related to the cross section for the scattering of transversely and longitudinally polarized $Z^{0}$ bosons. That is,

$$
\begin{aligned}
F_{T, L}^{\mathrm{NC}}\left(x, Q^{2}\right)= & \frac{Q^{2}}{4 \pi^{2} \alpha_{Z}} \int d^{2} \boldsymbol{r} \\
& \times \int_{0}^{1} d z\left|\psi_{T, L}^{Z^{0}}\left(z, \boldsymbol{r}, Q^{2}\right)\right|^{2} \sigma_{\text {dip }}(x, \boldsymbol{r}),
\end{aligned}
$$

where $\boldsymbol{r}$ denotes the transverse size of the color dipole, $z$ is the longitudinal momentum fraction carried by a quark, and $\psi_{T, L}^{Z}$ are the light-cone wave functions for the virtual $Z^{0}$ bosons with transverse or longitudinal polarizations. The small- $x$ neutrino structure function $F_{2}^{\nu N}$ is computed from expressions above taking $F_{2}=F_{T}+F_{L}$. Explicit expressions for the light-cone wave functions in the charged current case can be found in Refs. [10,11]. The corresponding expressions for the special case of massless quarks and considering an isoscalar target are presented in Ref. [12]. We quote Refs. [1,13,14] for the recent phenomenology using the color dipole picture in the case of $\mathrm{CC}$ neutrino interactions.

For the $Z^{0}$ boson, the coupling to quarks contains both vector and axial-vector parts and it is convenient to consider the basis of helicity spinors to decompose the $Z^{0}$ light-cone wave function into distinct vector and axialvector parts [15]:

$$
\Psi_{\lambda}^{\lambda_{1} \lambda_{2}}\left(\boldsymbol{r}, z, Q^{2}\right)=V_{\lambda}^{\lambda_{1} \lambda_{2}}\left(\boldsymbol{r}, z, Q^{2}\right)-A_{\lambda}^{\lambda_{1} \lambda_{2}}\left(\boldsymbol{r}, z, Q^{2}\right),
$$

where $\lambda_{1}$ and $\lambda_{2}$ denote the quark helicities. The probability densities of quark-antiquark states $\left|\Psi_{L}^{Z^{0}}\right|^{2}$ and $\left|\Psi_{T}^{Z^{0}}\right|^{2}$ are given by

$$
\left|\Psi_{L}^{Z^{0}}\right|^{2}=\sum_{\lambda_{1}, \lambda_{2}} \Psi_{0}^{\lambda_{1} \lambda_{2}}\left(\Psi_{0}^{\lambda_{1} \lambda_{2}}\right)^{*}=\sum_{\lambda_{1}, \lambda_{2}}\left(\left|V_{0}^{\lambda_{1} \lambda_{2}}\right|^{2}+\left|A_{0}^{\lambda_{1} \lambda_{2}}\right|^{2}\right),
$$

$$
\begin{aligned}
\left|\Psi_{T}^{Z^{0}}\right|^{2}= & \frac{1}{2}\left[\left|\Psi_{+1}^{+1 / 2+1 / 2}\right|^{2}+\left|\Psi_{+1}^{-1 / 2+1 / 2}\right|^{2}+\left|\Psi_{-1}^{-1 / 2-1 / 2}\right|^{2}\right. \\
& \left.+\left|\Psi_{-1}^{-1 / 2+1 / 2}\right|^{2}\right]
\end{aligned}
$$

The vector and axial-vector parts of the $Z^{0}$ wave function are given by [15]

$$
\begin{aligned}
& \left.V_{ \pm 1}^{\lambda_{1} \lambda_{2}}=-\sqrt{\alpha_{Z}} g_{V}^{f} \sqrt{2 N_{c}}\left\{ \pm i e^{ \pm i \theta_{r}[z} \delta_{\lambda_{1}, \pm} \delta_{\lambda_{2}, \mp}-(1-z) \delta_{\lambda_{1}, \mp} \delta_{\lambda_{2}, \pm}\right] \partial_{r}+m_{f} \delta_{\lambda_{1}, \pm} \delta_{\lambda_{2}, \pm}\right\} \frac{K_{0}(\varepsilon r)}{2 \pi}, \\
& V_{0}^{\lambda_{1} \lambda_{2}}=\sqrt{\alpha_{Z}} g_{V}^{f} \frac{\sqrt{N_{c}}}{Q} \delta_{\lambda_{1},-\lambda_{2}} 2 Q^{2} z(1-z) \frac{K_{0}(\varepsilon r)}{2 \pi}, \\
& \left.A_{ \pm 1}^{\lambda_{1} \lambda_{2}}=\sqrt{\alpha_{Z}} g_{A}^{f} \sqrt{2 N_{c}}\left\{-i e^{ \pm i \theta_{r}\left[z \delta_{\lambda_{1}, \pm}\right.} \delta_{\lambda_{2}, \mp}+(1-z) \delta_{\lambda_{1}, \mp} \delta_{\lambda_{2}, \pm}\right] \partial_{r} \pm m_{f}(1-2 z) \delta_{\lambda_{1}, \pm} \delta_{\lambda_{2}, \pm}\right\} \frac{K_{0}(\varepsilon r)}{2 \pi} \text {, } \\
& A_{0}^{\lambda_{1} \lambda_{2}}=\sqrt{\alpha_{Z}} g_{A}^{f} \frac{\sqrt{N_{c}}}{Q}\left\{\delta_{\lambda_{1},-\lambda_{2}} 2 \lambda_{1}\left[2 Q^{2} z(1-z)+2 m_{f}^{2}\right]-i \delta_{\lambda_{1}, \lambda_{2}} e^{-i 2 \lambda_{1} \theta_{r}} 2 m_{f} \partial_{r}\right\} \frac{K_{0}(\varepsilon r)}{2 \pi},
\end{aligned}
$$

where $\alpha_{Z}=4 \pi \alpha_{\text {em }} / \sin ^{2}\left(2 \theta_{W}\right)$ and $\varepsilon^{2}=z(1-z) Q^{2}+m_{f}^{2}$. The axial-vector couplings are given by $g_{V}^{f}=\sqrt{\rho}\left(I_{3}^{f}-\right.$ $\left.2 Q^{f} \sin ^{2} \theta_{W}\right)$ and $g_{A}^{f}=\sqrt{\rho}\left(I_{3}^{f}\right)$. Here, $I_{3}^{f}$ and $Q^{f}$ are the weak isospin and electromagnetic charge of quark $f$, respectively. 
The relative coupling strength of the neutral to charged current interactions is labeled by $\rho$, where $\rho=1$ at tree level in the

standard model. The weak mixing parameter, $\sin ^{2} \theta_{W}$, is related at tree level to $G_{F}, M_{Z}$, and $\alpha_{\text {em }}$ by $\sin ^{2} \theta_{W}=$ $4 \pi \alpha_{\mathrm{em}} / \sqrt{2} G_{F} M_{Z}^{2}$.

On the other hand, the dipole hadron cross section $\sigma_{\text {dip }}$ contains all information about the target and the strong interaction physics. Here, we consider two representative models for the dipole cross section. In what follows one takes the phenomenological parametrizations: (a) the Golec-Biernat-Wüsthoff model (GBW) [16] and (b) the impact parameter color glass condensate model ( $b$-CGC) model [17]. Both models are able to describe experimental data on inclusive and diffractive deep inelastic ep scattering at small- $x$. The $b$-CGC model is also successful in describing data on exclusive vector meson production at Deutsches Elektronen-Synchrotron-Hadron Electron Ring Accelerator (DESY-HERA) and includes important aspects such as impact parameter dependence, extended geometric scaling, and saturation physics at very small $x$. The idea behind it is to introduce the impact parameter dependence into the CGC model [18], where the dipole cross section is given by

$$
\sigma_{\text {dip }}(x, r)=2 \int d^{2} b N(x, r, b) \text {, }
$$

$N(x, r, b)= \begin{cases}\mathcal{N}_{0}\left(\frac{r Q_{\text {sat }}}{2}\right)^{2\left[\gamma_{s}+(1 / \kappa \lambda Y) \ln \left(2 / r Q_{\text {sat }}\right)\right]}: & r Q_{\text {sat }} \leq 2, \\ 1-e^{-A \ln ^{2}\left(B r Q_{\text {sat }}\right)}: & r Q_{\text {sat }}>2,\end{cases}$

where $Y=\ln (1 / x)$ and $Q_{\text {sat }}(x, b)$ is the impact parameter saturation scale defined as [17]

$$
Q_{\text {sat }}(x, b)=\left(\frac{x_{0}}{x}\right)^{\lambda / 2}\left[\exp \left(-\frac{b^{2}}{2 B_{\mathrm{CGC}}}\right)\right]^{1 / 2 \gamma_{s}}
$$

In the $b$-CGC model the evolution effects are included using an approximate solution to the Balitsky-Kovchegov equation. In particular, one has the slope $B_{\mathrm{CGC}}=$ $5.5 \mathrm{GeV}^{-2}$. The constants $A$ and $B$ are obtained from continuity conditions at $r Q_{\mathrm{sat}}=2$. We quote the original papers for details on the parametrizations and determination of their phenomenological parameters. Two important parameters are $\lambda$ and $\gamma_{s}$ (saturation anomalous dimension), which determine the energy dependence of the saturation scale $Q_{\text {sat }}$. Here, we use an effective light quark mass $m_{f}=$ $0.14 \mathrm{GeV}$ and the charm (bottom) mass is set to be $m_{c}=$ $1.4 \mathrm{GeV}\left(m_{b}=4.5 \mathrm{GeV}\right)$. In the dipole cross section we use $x=x_{B j}\left[1+\left(4 m_{f}^{2} / Q^{2}\right)\right]$.

For scattering on nuclei, we use the extension of the color approach for nuclear targets taking the GlauberGribov picture [9], without any new parameter. In this approach, the nuclear version is obtained replacing the dipole-nucleon cross section by the nuclear one,

$$
\begin{aligned}
\sigma_{\text {dip }}^{\text {nucleus }}(x, r ; A)= & 2 \int d^{2} b\{1 \\
& \left.-\exp \left[-\frac{1}{2} T_{A}(b) \sigma_{\text {dip }}^{\text {nucleon }}(x, r)\right]\right\},
\end{aligned}
$$

where $b$ is the impact parameter of the center of the dipole relative to the center of the nucleus and the integrand gives the total dipole-nucleus cross section for a fixed impact parameter. The nuclear profile function is labeled by $T_{A}(b)$ [19].

In Fig. 1, the structure functions $F_{2}^{\mathrm{NC}}\left(x, Q^{2}\right)$ (left panel) and $F_{L}^{\mathrm{NC}}\left(x, Q^{2}\right)$ (right panel) are presented as a function of the Bjorken variable $x$ for distinct boson virtualities using the $b$-CGC model. It is found that the $x$ dependence is approximately powerlike with a effective power which growths on $Q^{2}, \lambda=\lambda\left(Q^{2}\right)$. Namely, it goes from $\lambda\left(Q^{2}=\right.$ $\left.1 \mathrm{GeV}^{2}\right) \simeq 0.12$ up to $\lambda\left(Q^{2}=M_{Z}^{2}\right) \simeq 0.224$. Similar behavior is verified for the $F_{L}$ structure function, where a flattening is seen at very small $x$. This is explained by the QCD evolution which is reproduced in the $b$-CGC model due to a running effective anomalous dimension. It is interesting to call attention to the unusual behavior of the structure function in the limit of large $Q^{2}$ and large $x$, which is more evident for $F_{L}$. In order to estimate the uncertainty from the theoretical side, we compute the structure function using the simple GBW saturation model, which is presented in Fig. 2. The GBW model does not include the QCD evolution in the dipole cross section and in principle it is not suitable for very large virtualities. However, the effective power is similar to the $b$-CGC model with $F_{L}$ being distinct at $Q^{2}=M_{Z}^{2}$ (it could be a consequence of missing QCD evolution in the dipole cross section). It seems also that the flattening in $F_{L}$ is stronger in $b$-CGC than in GBW.
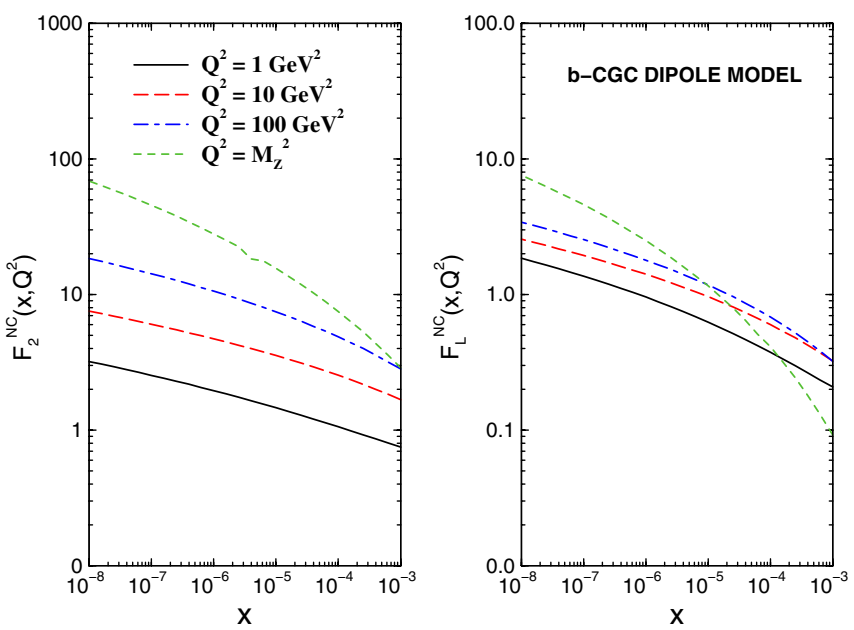

FIG. 1 (color online). The structure functions $F_{2}^{\mathrm{NC}}\left(x, Q^{2}\right)$ and $F_{L}^{\mathrm{NC}}\left(x, Q^{2}\right)$ as a function of $x$ for distinct boson virtualities (see text) using the $b$-CGC model. 

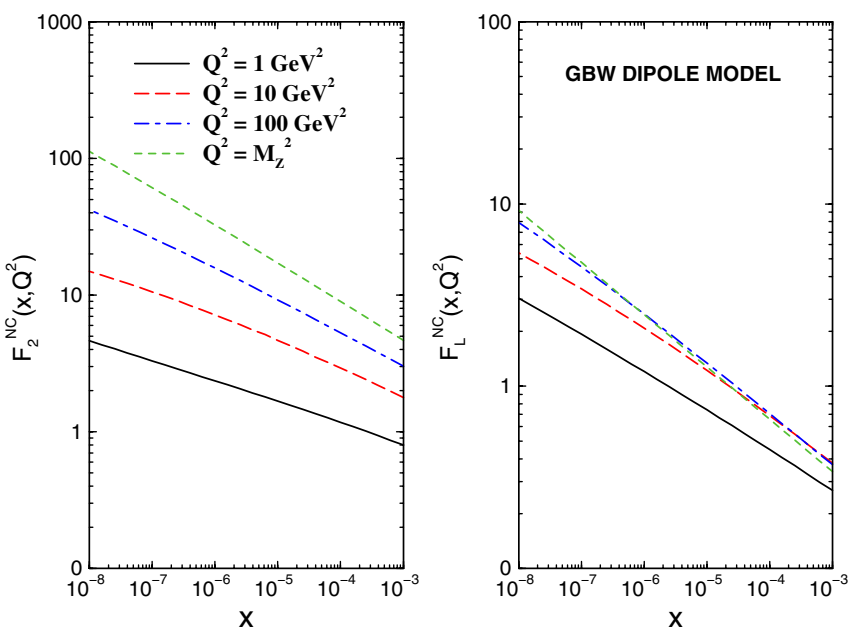

FIG. 2 (color online). The structure functions $F_{2}^{\mathrm{NC}}\left(x, Q^{2}\right)$ and $F_{L}^{\mathrm{NC}}\left(x, Q^{2}\right)$ as a function of $x$ for distinct boson virtualities (see text) using the GBW model.

In Fig. 3 we present the structure function $F_{2}$ as a function of $Q^{2}$ for fixed $x$. In the left panel one has the virtuality dependence for $b$-CGC and GBW models for two representative values of $x\left(x=10^{-2}\right.$ and $\left.x=10^{-6}\right)$. It is verified that the results show the usual logarithmic dependence on $Q^{2}$. The dipole models reproduce the scaling violations for the structure functions. There is a small deviation on the overall normalization (a few percent), which is more sizable at large $Q^{2}$. In the right panel, one has the flavor contribution to structure function. For the sake of illustration we consider the GBW model and $x=$ $10^{-3}$. For light quarks, the $(d, s)$ contribution is dominant over the $u$ quark, which is evident from the comparison among their electroweak couplings. The charm contribution corresponds to $13-14 \%$ of the total result at this
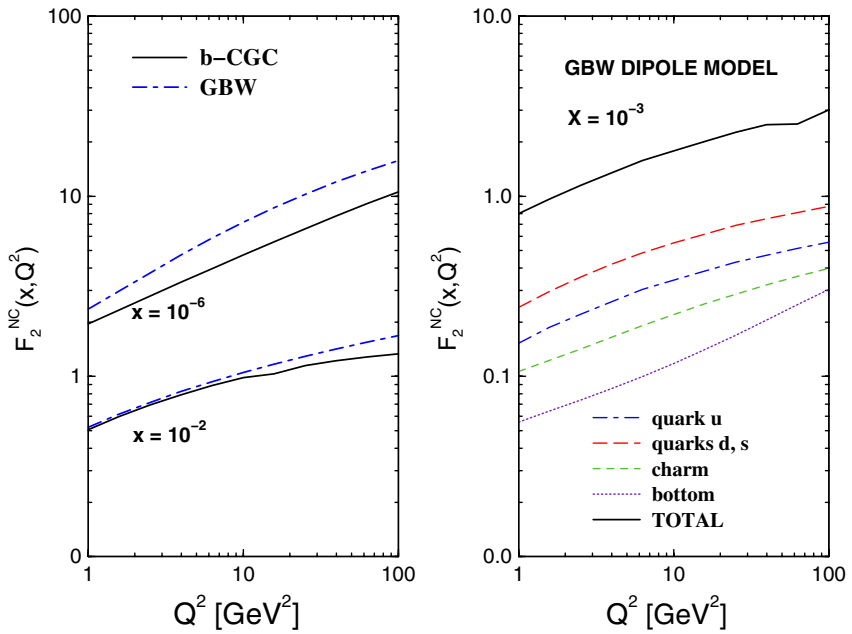

FIG. 3 (color online). The structure function $F_{2}^{\nu N}\left(x, Q^{2}\right)$ as a function $Q^{2}$ for both models (left panel) and its flavor contribution (right panel). $x$ value. The bottom contribution is very small, suppressed by its larger mass.

An important feature of neutral current neutrino-nucleon interaction within the color dipole picture is the geometric scaling property. Such a scaling is a basic property of the saturation physics. It means that the total $\gamma^{*} p$ cross section at large energies is not a function of the two independent variables $x$ and $Q$, but is rather a function of the single variable $\tau_{p}=Q^{2} / Q_{\text {sat }}^{2}(x)$ as shown in Refs. [20,21]. That is, $\sigma_{\gamma^{*} p}\left(x, Q^{2}\right)=\sigma_{\gamma^{*} p}\left(\tau_{p}\right)$. Similar scaling is predicted for lepton-nucleus scattering at high energies [22]. In Ref. [1] we have shown that scaling is present in charged current neutrino scattering. It is exact for light quarks, whereas is partially violated by charm contributions $c \bar{s}$ and $s \bar{c}$ due to different quark masses. We can qualitatively show the geometric scaling property in the NC neutrino-nucleon scattering by writing down the light-cone wave functions (for sake of simplicity, we take $g_{V} / g_{A} \approx 1$ ):

$$
\begin{aligned}
\left|\Psi_{T}^{Z^{0}}\right|^{2} \propto[ & \left.(1-z)^{2}+z^{2}\right] m_{f}^{2} K_{0}^{2}(\varepsilon r) \\
& +\left[(1-z)^{2}+z^{2}\right] \varepsilon^{2} K_{1}^{2}(\varepsilon r),
\end{aligned}
$$

$$
\begin{aligned}
\left|\Psi_{L}^{Z^{0}}\right|^{2} \propto & {\left[4 Q^{4} z^{2}(1-z)^{2}+\mid\left(2 Q^{2} z(1-z)+2 m_{f}^{2}\right)^{2}\right] } \\
& \times \frac{K_{0}^{2}(\varepsilon r)}{Q^{2}}+\left[4 m_{f}^{2} \varepsilon^{2}\right] \frac{K_{1}^{2}(\varepsilon r)}{Q^{2}}
\end{aligned}
$$

The equations above are different from the usual expressions for light-cone wave functions in electron-proton DIS even in the massless case due to the distinct weak couplings. Neglecting the quark mass, one can rewrite Eq. (1) as

$$
\begin{aligned}
\sigma_{T,(L)}\left(Z^{0}+N \rightarrow X\right) \propto & \int d z \int \bar{r} d \bar{r} f_{T,(L)}(z) K_{1,(0)}^{2} \\
& \times(\sqrt{z(1-z)} \bar{r}) \sigma_{\text {dip }}\left(\frac{Q_{\text {sat }}^{2}}{Q^{2}} \bar{r}\right),
\end{aligned}
$$

where we have $f_{T}(z)=\left[(1-z)^{2}+z^{2}\right](1-z) z$ and $f_{L}(z)=z^{2}(1-z)^{2}$. Here, we rescaled the transverse size $r$ to the dimensionless variable $\bar{r}=Q r$ and suppose a scaling behavior for the dipole cross section, $\sigma_{\text {dip }}=$ $\sigma_{\text {dip }}\left(Q_{\text {sat }} r\right)$. This last assumption is fulfilled by several saturation approaches, as for the GBW model where $\sigma_{\text {dip }}=\sigma_{0}\left[1-\exp \left(-r^{2} Q_{\text {sat }}^{2} / 4\right)\right]$. Therefore, we have a scaling appearing in the $\mathrm{NC}$ boson-nucleon cross section, $\sigma_{\text {tot }}\left(Z^{0}+N \rightarrow X\right)=\sigma_{\text {tot }}\left(\tau_{p}=Q_{\text {sat }}^{2} / Q^{2}\right)$. Similar scaling should be present in the nuclear case, as shown in Ref. [1].

In Fig. 4 we present the study for nuclear targets. The structure function $F_{2}^{\mathrm{NC}}$ is presented as a function of $x$ for fixed values of virtualities $\left(Q^{2}=1 \mathrm{GeV}^{2}\right.$ and $Q^{2}=$ $50 \mathrm{GeV}^{2}$ ). We select the following nuclei: carbon, aluminum, iron, and lead. We stress that for the NuSonG experiment we will have an isoscalar target similar to $A=\mathrm{Fe}$ as for $\mathrm{NuTeV}$. The nuclear shadowing is more intense for heavy nuclei, smaller $x$, and virtualities. In particular, for 

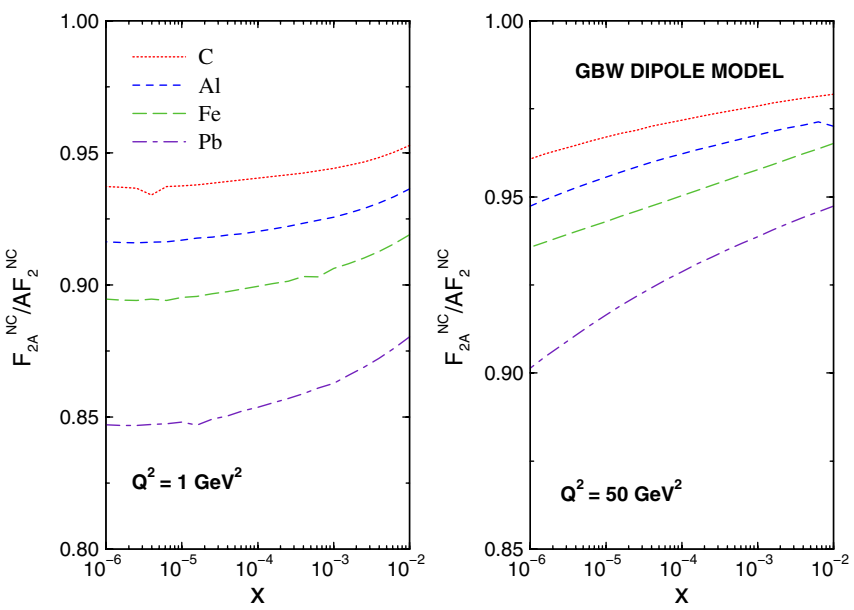

FIG. 4 (color online). The nuclear ratio $R_{A}=F_{2 A}^{\mathrm{NC}} / A F_{2}^{\mathrm{NC}}$ as a function of the Bjorken variable for fixed virtualities $\left(Q^{2}=1\right.$ and $50 \mathrm{GeV}^{2}$ ) and several nuclear targets.

iron targets the nuclear shadowing is of order $10 \%$ at $Q^{2}=$ $1 \mathrm{GeV}^{2}$, whereas it reaches to $15-20 \%$ for lead. Nuclear shadowing is small at large virtualities even at low $x$. This fact is also verified in charged current interactions as investigated in Ref. [1], where the nuclear ratio has been computed for $F_{2}^{\mathrm{CC}}$ and $x F_{3}$. Some questions are in order here. The information on isospin violation is quite relevant for electroweak measurements and isospin symmetry is assumed in the NuTeV analysis [6]. Violation of isospin symmetry can be one important source of the $\mathrm{NuTeV}$ anomaly. Therefore, better constraint on the isospin violation will be crucial to the interpretation of new neutrino experiments. The NuSOnG experiment [7] purposes to measure separately $\Delta F_{2} \equiv \frac{5}{18} F_{2}^{\mathrm{CC}}\left(x, Q^{2}\right)-F_{2}^{\mathrm{NC}}\left(x, Q^{2}\right)$ in the $\mathrm{CC} W^{ \pm}$exchange and the $\mathrm{NC} \gamma / Z$ exchange processes, which can also constrain charge symmetry violation. As $\mathrm{NuSOnG}$ (glass target, $\mathrm{SiO}_{2}$, is very nearly isoscalar) could measure $F_{2}^{\mathrm{CC}}$ on a variety of targets, this will reduce the systematics associated with the heavy nuclear target corrections providing new information on the isospin violation. In particular, for $\mathrm{SiO}_{2}$ we have $Z_{\mathrm{O}}=8, Z_{\mathrm{Si}}=14$, $m_{\mathrm{O}}=15.994$, and $m_{\mathrm{Si}}=28.0855$. Using $A=Z+N$ we have $(N-Z) / A=(A-2 Z) / A$, which yields $(N-$ $Z) / A \sim-0.000375$ for $\mathrm{O}$ and $(N-Z) / A \sim 0.00304$ for $\mathrm{Si}$. Therefore, since NuSOnG will provide high statistics $F_{2}^{\mathrm{CC}}$ measurements for a variety of $A$ targets, this will yield an alternate handle on the isospin violation and will also improve our understanding of the associated nuclear corrections.

Having discussed the main features of our results for structure functions and associated nuclear shadowing, it is timely to provide a more detailed and quantitative discussion with respect to existing data and/or expected results. In the charged current case, the present approach has successfully described [1] the existing data (CCFR and $\mathrm{NuTeV}$ ) for structure functions $F_{2}$ and $F_{1}$ as well as the quantity $\Delta x F_{3}$ in the small- $x$ regime. We verified for the first time in literature an approximated geometric scaling property on CCFR and NuTeV low- $x$ data. From a theoretical point of view, the geometric scaling is slightly broken in the charge current case due to the different quark masses appearing in Eqs. (3) and (4) (this is evident in the $c \bar{s} / \bar{c} s$ contribution to wave functions). In the present work we focus on the neutral current interactions, where geometric scaling should be observed in future experimental measurements. Most of the current and future experiments, as Miner $\nu$ a, are dedicated to charged current interactions. The NuSOnG experiment will allow one to measure neutral current interactions with a large number of DIS events, which can be used to measure six structure functions (three on neutrinos and three for antineutrinos) as well as the strange quark distributions. The range in $x$ and $Q^{2}$ on this experiment is similar to the $\mathrm{NuTeV}$ domain, that is, $1<$ $Q^{2}<100 \mathrm{GeV}^{2}$ and $10^{-4}<x<0.1$. It will be able to provide high statistics DIS measurements across a wide $x$ range. Therefore, the color dipole approach can be safely used to describe low- $x$ NuSOnG data. The statistics on this experiment will be sufficient to distinguish theoretical uncertainties associated with model dependence. In our case, this feature allows one to disentangle models at large- $Q^{2}$ bins, where QCD evolution is increasingly important in the dipole cross sections. NuSOnG will improve by 1 order of magnitude the number of events compared to $\mathrm{NuTeV}$ and CCFR, the previous highest statistics experiments. As an example, with the high statistics of NuSOnG, precise measurement of $R_{L}$ (the longitudinal to transverse virtual boson absorption cross-section ratio) will be possible from neutrino scattering for the first time. Thus, we could confront our theoretical predictions for the longitudinal structure function.

In the present work we take into account explicitly the heavy quark contributions (charm and bottom) to the structure functions. Notice that the heavy quark production mechanism is different in the $\mathrm{CC}$ and $\mathrm{NC}$ processes, where the distinct threshold kinematics behaviors have to be implemented. In the color dipole approach this characteristic is automatically present in a flavor-dependent dipole cross section. In addition, the dipole approach has a strong advantage in contrast to the usual partonic description of neutrino interactions: it allows for a smooth transition between low to large virtualities. Namely, the dipole formalism takes into account higher twist contributions whereas the usual perturbative QCD calculations are not valid anymore in this kinematical regime. The advantage is evident also in the implementation of nuclear shadowing. The usual partonic pQCD approach uses nuclear ratios (and therefore information on gluon shadowing) extracted phenomenologically from data, where nuclear shadowing has a very large uncertainty. In the dipole approach, nuclear shadowing is dynamically implemented and the theoretical uncertainties are less dramatic. The main quantity which drives the shadowing corrections is the nuclear saturation scale, $Q_{\text {sat }, A}(x)$. As a simplification, we can write 
$Q_{\text {sat }, A}^{2}=A^{1 / 3} Q_{\text {sat }, p}^{2}$, where $Q_{\text {sat }, p}$ is the saturation scale for a nucleon target $\left(Q_{\text {sat, } p} \approx 1 \mathrm{GeV}\right.$ for $\left.x=10^{-4}\right)$. As NuSOnG is planned for several nuclear targets, it will allow for careful studies on the parton saturation phenomenon. For instance, in a lead target the saturation scale is enhanced by a factor of 6 in contrast to the proton target.

Let us compare now our calculation with other small- $x$ approaches in literature. First of all, notice that the present study in the dipole approach is the first one which considers the correct heavy quark content and nuclear shadowing implementation. Our investigation is similar to work on Ref. [23], where the same dipole formalism is considered. However, in that work the quark masses are considered to be zero, $m_{f}=0$, which is suitable only for ultrahigh energy cosmic neutrinos. In addition, nuclear shadowing corrections for nuclear targets are not explicitly included as in our case and theoretical uncertainties are not addressed. Our results are consistent in addition to the unified Dokshitzer-Gribov-Lipatov-Altarelli-Parisi/Balitsky-

Fadin-Kuraev-Lipatov calculations presented in Ref. [24], where the semihard factorization approach is considered. At leading logarithmic approximation, the color dipole formalism is equivalent to such an approach. In Ref. [24] nuclear shadowing is not considered as the cross section is calculated for nucleon targets. Concerning our studies on nuclear shadowing, it should be noticed that the GlauberGribov formalism has been used. There are other options suitable for the dipole approach (we quote Ref. [25] for a review). One of them is the CGC formalism, where the small- $x$ partons (slow gluons) are treated classically due to the high occupation number $\propto 1 / \alpha_{s}$. This number is as high as it can be and thus this field is often referred to as saturation physics. The squared saturation momentum or saturation scale is given by $Q_{\text {sat }}^{2} \propto A T_{A}(b) x G\left(x, Q^{2}\right)$, where $x G$ is the gluon distribution on proton. In general, the model based on Gribov inelastic shadowing gives a larger shadowing than CGC implementations.

As a final study, we compute the associated charm production in NC neutrino-nucleus interactions, $\sigma_{\mathrm{NC}}(c \bar{c}) / \sigma_{\mathrm{NC}}^{\mathrm{DIS}}$. The total NC neutrino-nucleon cross sections as a function of the neutrino energy and atomic number are given by the integration over available phase space and read as

$$
\sigma_{(\nu, \bar{\nu})}^{\mathrm{NC}}\left(E_{\nu} ; A\right)=\int_{Q_{\min }^{2}}^{s} d Q^{2} \int_{Q^{2} / s}^{1} d x \frac{1}{x s} \frac{\partial^{2} \sigma_{(\nu, \bar{\nu})}^{\mathrm{NC}}}{\partial x \partial y},
$$

where

$$
\begin{aligned}
\frac{\partial^{2} \sigma_{(\nu, \bar{\nu})}^{\mathrm{NC}}}{\partial x \partial y}= & \frac{G_{F}^{2} m_{N} E_{\nu}}{\pi}\left(\frac{m_{Z}^{2}}{Q^{2}+m_{Z}^{2}}\right)^{2} \\
& \times\left[\frac{1+(1-y)^{2}}{2} F_{2}^{\mathrm{NC}}\left(x, Q^{2} ; A\right)\right. \\
& \left.-\frac{y^{2}}{2} F_{L}^{\mathrm{NC}}\left(x, Q^{2} ; A\right)\right],
\end{aligned}
$$

where $G_{F}$ is the weak Fermi coupling constant, $m_{N}$ is the nucleon mass, $E_{\nu}$ is the incident neutrino energy, and $Q^{2}$ is the square of the four-momentum transfer to the nucleon. The variable $y=E_{\text {had }} / E_{\nu}$ is the fractional energy transferred to the hadronic vertex with $E_{\text {had }}$ being the measured hadronic energy, and $x=Q^{2} / 2 m_{N} E_{\nu} y$ is the Bjorken scaling variable (fractional momentum carried by the struck quark). The minimum $Q_{\min }^{2} \propto O(1) \mathrm{GeV}^{2}$ is introduced in order to stay in the DIS region. Here, one considers ultrahigh energy neutrinos, where the valence quark contribution stays constant and physics is driven by sea quark contributions. Hence, the $x F_{3}$ contribution should be negligible and it will be disregarded. A similar cross section can be computed for the charm contribution by replacing the respective charm structure function. We quote Refs. [26,27] for the previous analysis on CC and $\mathrm{NC}$ neutrino cross sections using the color dipole picture.

Recently, the CHORUS Collaboration [28] reported an extraction of the associated charm production in $\mathrm{NC} \mathrm{n}$ eutrino DIS at $E_{\nu}=27 \mathrm{GeV}$. The experimental determination found $\sigma(c \bar{c}) / \sigma_{\text {tot }}=3.62_{-2.42}^{+2.95}($ stat $) \pm$ 0.54 (syst) $\times 10^{-3}$ at the neutrino energy $E_{\nu}=27 \mathrm{GeV}$. We quote also previous determinations, as the E531 experiment [29] $\sigma(c \bar{c}) / \sigma_{\text {tot }}=1.3_{-1.1}^{+3.1} \times 10^{-3}$ for energy $E_{\nu}=22 \mathrm{GeV}$ and the $\mathrm{NuTeV}$ experiment [30] giving $\sigma(c \bar{c}) / \sigma_{\text {tot }}=6.4_{-4.6}^{+5.5} \times 10^{-3}$ at $E_{\nu}=154 \mathrm{GeV}$. We have found theoretically $\sigma(c \bar{c}) / \sigma_{\text {tot }}=2.7 \times 10^{-2}$ for $E_{\nu}=$ $27 \mathrm{GeV}$ and $\sigma(c \bar{c}) / \sigma_{\mathrm{tot}}=1.35 \times 10^{-1}$ for $E_{\nu}=$ $154 \mathrm{GeV}$. Our results are systematically above the experimental measurements, which can be explained by the extrapolation to very low energies of the color dipole results for charm contribution. It is well known that the dipole approaches overestimate the structure functions for $x \rightarrow 1$. At high energies our predictions are consistent with other approaches using small- $x$ formalisms $[23,24]$.

\section{COMMENTS AND CONCLUSIONS}

As a summary, an analysis of small- $x$ NC neutrinonucleus DIS is performed within the color dipole formalism. The structure functions $F_{2}^{\nu N}, F_{L}^{\nu N}$, and the nuclear ratio $R_{A}=F_{2}^{\nu A} / A F_{2}^{\nu N}$ are calculated. In order to investigate the theoretical uncertainties we employed two phenomenological parametrizations for the dipole cross section which successfully describe small- $x$ inclusive, diffractive $e p$ DIS data and exclusive production of vector mesons. Nuclear shadowing is taking into account through Glauber-Gribov formalism. We have found deviations among the models at very small $x$ and mostly at large virtualities. It is found that small- $x$ data show a geometric scaling property for the $\mathrm{NC}$ boson-hadron cross section as a function of the scaling variable $\tau$. Although the results presented here are compelling, further investigations are requested. In particular, it would be useful to compare the present calculations to measurements of the neutrinonucleus structure function in smaller values of $x$ than the 
currently measured in the accelerator experiments. This is the reason we have focused on the NuSonG purpose that will provide high statistics $F_{2}^{\mathrm{CC}}$ and $F_{2}^{\mathrm{NC}}$ measurements for a variety of nuclear targets. We also compute the charm content to the total $\mathrm{NC}$ neutrino cross section and we show that it is consistent with current experimental measurements. So, we have a description of small- $x$ structure functions in neutrino-proton interactions using a color dipole formalism. Despite the limitations of the current approach for the low energy neutrinos, our estimates are comparable to NC neutrino-nuclei data for charm production. Our calculation is also consistent with other approaches in literature in the high-energy limit.

\section{ACKNOWLEDGMENTS}

This work was supported by CNPq (Brazil).
[1] M. B. Gay Ducati, M. M. Machado, and M. V. T. Machado, Phys. Lett. B 644, 340 (2007).

[2] A. H. Mueller, Nucl. Phys. B335, 115 (1990); N. N. Nikolaev and B. G. Zakharov, Z. Phys. C 49, 607 (1991).

[3] W. G. Seligman et al. (CCFR Collaboration), Phys. Rev. Lett. 79, 1213 (1997).

[4] B. T. Fleming et al. (CCFR Collaboration), Phys. Rev. Lett. 86, 5430 (2001).

[5] U. K. Yang et al., Phys. Rev. Lett. 86, 2742 (2001).

[6] M. Tzanov et al. (NuTeV Collaboration), Phys. Rev. D 74, 012008 (2006).

[7] T. Adams et al. (NuSOnG Collaboration), Int. J. Mod. Phys. A 24, 671 (2009); J. M. Conrad, AIP Conf. Proc. 981, 243 (2008).

[8] E. A. Paschos and L. Wolfenstein, Phys. Rev. D 7, 91 (1973).

[9] N. Armesto, Eur. Phys. J. C 26, 35 (2002).

[10] V. Barone, M. Genovese, N. N. Nikolaev, E. Predazzi, and B. G. Zakharov, Phys. Lett. B 292, 181 (1992).

[11] V. Barone, M. Genovese, N. N. Nikolaev, E. Predazzi, and B. G. Zakharov, Phys. Lett. B 328, 143 (1994).

[12] K. Kutak and J. Kwieciński, Eur. Phys. J. C 29, 521 (2003).

[13] R. Fiore and V. R. Zoller, JETP Lett. 82, 385 (2005).

[14] R. Fiore and V. R. Zoller, Phys. Lett. B 632, 87 (2006).

[15] L. Motyka and G. Watt, Phys. Rev. D 78, 014023 (2008).
[16] K. Golec-Biernat and M. Wüsthoff, Phys. Rev. D 59, 014017 (1998); 60, 114023 (1999).

[17] G. Watt and H. Kowalski, Phys. Rev. D 78, 014016 (2008).

[18] E. Iancu, K. Itakura, and S. Munier, Phys. Lett. B 590, 199 (2004).

[19] C. W. De Jager, H. De Vries, and C. De Vries, At. Data Nucl. Data Tables 14, 479 (1974).

[20] A. M. Staśto, K. Golec-Biernat, and J. Kwieciński, Phys. Rev. Lett. 86, 596 (2001).

[21] V. P. Goncalves and M. V. T. Machado, Phys. Rev. Lett. 91, 202002 (2003).

[22] N. Armesto, C. A. Salgado, and U. A. Wiedemann, Phys. Rev. Lett. 94, 022002 (2005).

[23] K. Kutak and J. Kwieciński, Eur. Phys. J. C 29, 521 (2003).

[24] J. Kwieciński, A. D. Martin, and A. M. Staśto, Phys. Rev. D 59, 093002 (1999).

[25] N. Armesto, J. Phys. G 32, R367 (2006).

[26] M. V. T. Machado, Phys. Rev. D 71, 114009 (2005).

[27] M. V. T. Machado, Phys. Rev. D 70, 053008 (2004).

[28] A. Kayis-Topaksu et al. (CHORUS Collaboration), Eur. Phys. J. C 52, 543 (2007).

[29] N. Ushida et al. (E531 Collaboration), Phys. Lett. B 206, 375 (1988).

[30] M. Goncharov et al. (NuTeV Collaboration), Phys. Rev. D 64, 112006 (2001). 